\title{
Magnetic composites based on NR and strontium ferrite
}

\author{
Ján Kruželák ${ }^{a}$, Andrea Kvasničákováa, \\ Rastislav Dosoudil ${ }^{\mathrm{b}}$, Ivan Hudec ${ }^{\text {a }}$ \\ ${ }^{a}$ Department of Plastics, Rubber and Fibres, Faculty of Chemical and Food Technology, \\ Slovak University of Technology in Bratislava, Radlinského 9, 81237 Bratislava, Slovakia \\ ${ }^{b}$ Department of Electromagnetic Theory, Faculty of Electrical Engineering and Information Technology, \\ Slovak University of Technology in Bratislava, Il'kovičova 3, 812 19, Bratislava, Slovakia \\ jan.kruzelak@stuba.sk
}

\begin{abstract}
Two types of composites based on natural rubber (NR) and strontium ferrite were tested in this study. Composites of the first type were prepared by incorporation of strontium ferrite in the concentration range ranging from 0 to $100 \mathrm{phr}$ (parts per hundred rubber) into pure NR based rubber matrix, while with those of the second type, strontium ferrite was dosed in the same concentration level into NR based rubber batch with constant amount of carbon black -25 phr. For rubber matrices cross-linking, a standard sulfur based curing system was used. This work is focused on the effect of magnetic filler content on physico-mechanical, magnetic and thermo-physical properties of composite materials. Subsequently, the cross-link density and the structure of the formed sulfidic cross-links were examined. The results showed that the cross-link density of both types of composites increased with the increasing content of magnetic filler, while the structure of the sulfidic cross-links was almost not influenced by the amount of strontium ferrite. Tensile strength of rubber composites with pure rubber matrix was slightly improved by the incorporation of ferrite, while in case of composites based on a carbon black batch, the incorporation of magnetic filler resulted in the decrease of this characteristic. The presence of magnetic filler in both types of composites leads to a significant increase of the remanent magnetic induction.
\end{abstract}

Keywords: rubber composite, magnetic filler, cross-link density, tensile strength, remanent magnetic induction

\section{Introduction}

Ferrite permanent magnets of the general formula $\mathrm{SrFe}_{12} \mathrm{O}_{19}$ or $\mathrm{BaFe}_{12} \mathrm{O}_{19}$ contain approximately $80 \%$ of iron oxide and $20 \%$ of strontium or barium. They are a class of technologically important magnetic materials with high values of magneto-crystalline anisotropy as well as magnetic characteristics (mainly coercivity and remanent magnetic induction) (Wang at al., 2009; Namai at al.; Li at al., 2015). Moreover, they exhibit good chemical stability with high resistance to solvents, salts, lubricants, alkalis, harmful gases, soft acids and atmospheric effects. Operating temperature of ferrites ranges between $-40{ }^{\circ} \mathrm{C}$ to $+250{ }^{\circ} \mathrm{C}$. These outstanding properties include ferrites in important magnetic materials which cannot be easily replaced. Magnetic properties of ferrites for specific applications can be adapted by selection of components, composition, by supplying suitable metal ions and by other suitable techniques (Lechevallier and LeBreton, 2005; Xie at al., 2013; Pullar, 2012).

Incorporation of magnetic crystalline ferrites into various rubber matrices leads to the preparation of magnets known as ferrite rubber magnets (Pattanayak at al., 2016; Gutiérrez at al., 2015; Jung at al., 2016; Bellucci at al., 2016; Sýkora at al., 2016). Rubber magnetic composites are materials consisting of at least two phases: magnetic powder as filler and continuous rubber matrix. An important advantage of these materials is they can be prepared and processed by technologies typically used for polymer composites processing. They can be bent, coiled and shaped without the loss of their magnetic characteristics. Additionally, they are very resistant to corrosion and therefore no surface treatment is necessary. Due to their elasticity and easy processability, they are suitable for applications in which good elasticity and high flexibility are required. These attributes in combination with unique magnetic characteristics rank them among progressive developing materials which have already found the utilization in microwave and radar technology, vibration absorbers, motor parts, memory devices, variable impedance surfaces, inductor cores, sensors of magnetic and electromagnetic fields and in many other technological applications (Vinayasree at al., 2016; Tong at al., 2013; Das at al., 2015).

In this work, rubber magnetic composites were prepared by the incorporation of strontium ferrite into NR based pure rubber matrix or carbon black batch. The main aim was to observe the influence of magnetic filler content on physico-mechanical, magnetic and thermo-physical properties of the tested composites. Cross-link density and structure of the formed cross-links were also investigated. 


\section{Experimental}

\section{Materials}

Natural rubber (NR), type SMR20 (Mardec, Malaysia), and carbon black batch based on natural rubber, type SMR 20 (content of carbon black N330 - 25 phr, Elastorsa, Martin, SR), were used as rubber matrices. Strontium ferrite, $\mathrm{SrFe}_{12} \mathrm{O}_{19}$, type FD 8/24, supplied as magnetic filler by Magnety, Světlá Hora, Czech Republic, was dosed to the rubber formulations in the concentration range from 0 to 100 phr. Characteristics of the applied strontium ferrite are provided in Tab. 1. For rubber matrices cross-linking, a standard semi EV-sulfur curing system consisting of zinc oxide $-3 \mathrm{phr}$ (Slovlak, Košeca, Slovakia), stearic acid -2 phr (Setuza, Ústí nad Labem, Czech Republic), N-Cyclohexyl2-benzothiazole sulfenamide CBS - 1.5 phr (Duslo, Šala, Slovakia), and sulfur - 1.3 phr (Siarkopol, Tarnobrzeg, Poland) was used. Phr stands for parts per hundred rubber.

Tab. 1. Characteristics of strontium ferrite.

\begin{tabular}{lc}
\hline Characteristics & Value \\
\hline Particle size $(\mu \mathrm{m})$ & $0.1-30$ \\
Specific surface area $\left(\mathrm{m}^{2} / \mathrm{g}\right)$ & 4.06 \\
Total porosity $(\%)$ & 55.62 \\
Total volume of pores $\left(\mathrm{cm}^{3} / \mathrm{g}\right)$ & 0.254 \\
Density $\left(\mathrm{g} / \mathrm{cm}^{3}\right)$ & 4.73 \\
Coercivity $(\mathrm{kA} / \mathrm{m})$ & 117 \\
Remanent magnetic induction $(\mathrm{T})$ & 0.17 \\
\hline
\end{tabular}

\section{Methods}

Preparation and curing of rubber compounds

Rubber compounds were prepared in a laboratory appliance Brabender in two mixing steps. Temperature of the chamber was set to $90{ }^{\circ} \mathrm{C}$ and the mixing process was carried out at the rotor speed of $50 \mathrm{rpm}$. In the first step, rubber and filler were compounded. Mixing of ingredients in the first step was performed for $9 \mathrm{~min}$ in the temperature range of $90-95^{\circ} \mathrm{C}$. Components of the curing systems were introduced in the second step ( $4 \mathrm{~min}, 90-95^{\circ} \mathrm{C}$ ). Then, the mixed rubber compounds were shaped into thin sheets by a two roll calender.

Curing characteristics were investigated from the corresponding curing isotherms measured on Rheometer Monsanto R100 at $150{ }^{\circ} \mathrm{C}$. The rubber compounds were cured at $150{ }^{\circ} \mathrm{C}$ with regard to their optimum cure time under the pressure of $15 \mathrm{MPa}$. Hydraulic press FONTIJNE was employed to carry out the curing process.

\section{Determination of cross-link density and cross-link structure}

Two different methods were used to determine the cross-link density of rubber composites.

Chemical cross-link density, $\nu_{c h}$, was determined based on equilibrium swelling of samples in xylene. First, the samples were swelled in xylene until equilibrium swelling was reached. The cross-link density was then calculated based on the equilibrium swelling degree by applying the Krause modified Flory-Rehner equation (1) for filled vulcanizates (Kraus, 1963):

$$
\nu_{c h}=-\frac{V_{r 0}}{V_{S}} \frac{\ln \left(1-V_{r}\right)+V_{r}+\chi V_{r}^{2}}{V_{r}^{1 / 3} V_{r 0}^{2 / 3}-0,5 V_{r}}
$$

$\nu_{c h}-$ cross-link density $\left(\mathrm{mol} / \mathrm{cm}^{3}\right), V_{r 0}-$ volume fraction of rubber in equilibrium swelling sample of vulcanizate in absence of fillers, $V_{r}$ - volume fraction of rubber in equilibrium swelling sample of filled vulcanizate, $V_{S}-$ molar volume of solvent $\left(\right.$ for xylene $\left.=123.45 \mathrm{~cm}^{3} / \mathrm{mol}\right), \chi-$ Huggins interaction parameter (for measuring conditions $\chi=$ 0.4106).

In the second method, based on deformation measurements, the total cross-link density, $\nu_{t}$, was calculated by means of the Mooney-Rivlin equation (Hamed, 1992) (2), utilizing relation (3):

$$
\frac{\sigma}{2\left(\alpha-\alpha^{-2}\right)}=C_{1}+\frac{C_{2}}{\alpha}
$$

$\sigma-$ tension $(\mathrm{MPa}), \alpha-$ relatively extension (\%), $C_{1}, C_{2}-$ constants,

$$
\nu_{\mathrm{t}}=2 C_{1} / R T
$$

$R=8.314 \mathrm{~J} / \mathrm{K} \cdot \mathrm{mol}, T=293.15 \mathrm{~K}$.

The measurements were carried out using an Inspekt desk $5 \mathrm{kN}$ apparatus (Metrotest) up to $100 \%$ deformation with the deformation velocity of $10 \mathrm{~mm} / \mathrm{min}$.

Cross-link structure of vulcanizates was determined using the thiol-amine method in argon atmosphere as described in (Saville and Watson, 1967; Morrison and Porter, 1984; Warner, 1994). Propane-2-thiol was used, which has the ability to decompose polysulfidic cross-links. First, the chemical cross-link density, $v_{c h}$, of original samples was determined. Then, the samples were treated with a solution of propane-2-thiol for two hours at laboratory temperature in argon atmosphere and the cross-link density was determined again $\left(v_{P T}-\right.$ the cross-link density of samples after propane-2-thiol treatment). The content of polysulfidic $S x$ was then calculated by equation (4) and combinations of disulfidic $S 2$ a monosulfidic $S$ cross-links.

$$
S x=\nu_{c h}-\nu_{P T}, S x=\left[\left(\nu_{c h}-\nu_{P T)} \cdot 100\right] / \nu_{c h}\right.
$$




\section{Determination of physico-mechanical properties}

Tensile properties of rubber magnetic composites were determined using a Zwick Roell/Z 2.5 appliance. The measurements were carried out in accordance with the valid technical standards at the laboratory temperature and cross-head speed of $500 \mathrm{~mm} / \mathrm{min}$. The tested composites were cut into double side dumbbell-shaped test specimens (width of $6.4 \mathrm{~mm}$, length of $8 \mathrm{~cm}$, thickness of $2 \mathrm{~mm}$ ). Hardness was measured using a durometer and the unit was expressed in Shore A.

\section{Determination of magnetic characteristics}

Magnetic characteristics of composites were evaluated using a magnetometer TVM-1 at the maximum coercivity of $H_{m}=750 \mathrm{kA} / \mathrm{m}$. The basic principle of measurement was the induction method of scanning of scattering magnetic flux, $\Phi$, induced by magnetic vibrating sample. Magnetic field is generated by two cores of a Weiss electromagnet at the minimum distance of poles adapters of $7.5 \mathrm{~mm}$. The induced tension proportional to magnetic flux time dependence in the sample is scanned with a system of four small cores. The cores eliminate the influence of electromagnet magnetic fields time instability and the change of magnetic flux is directly proportional to magnetic induction, $B$. Specimens for magnetic characteristics evaluation were of prism shape $(8 \times 4 \times 2 \mathrm{~mm})$.

\section{Evaluation of thermo-physical characteristics of composites}

An Isomet appliance was applied to evaluate the thermo-physical characteristics of prepared materials. The measurement is based on the analysis of time dependence of thermal response to the impulses of the thermal flow into the analyzed material. The thermal flow is generated by scat-

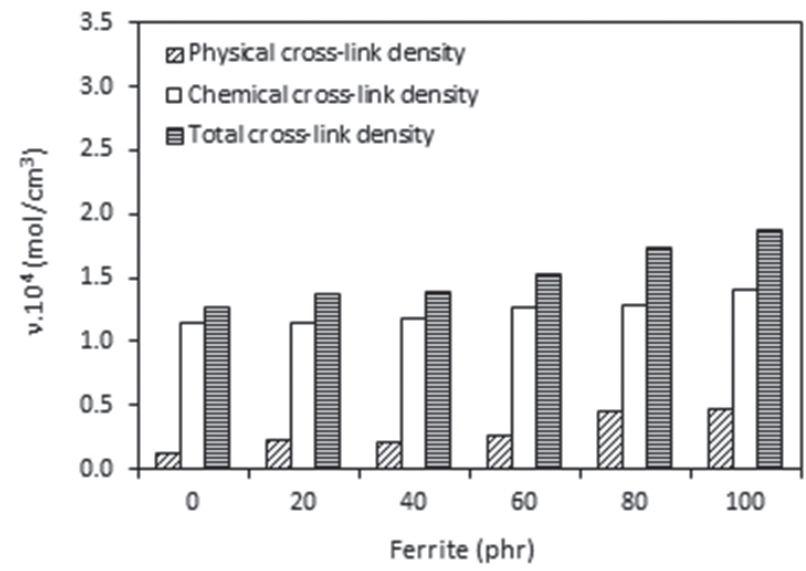

Fig. 1. Influence of ferrite content on physical $\nu_{p h}$, chemical $\nu_{c h}$ and total $\nu_{t}$ cross-link density of composites based on NR. tered electric discharge in a probe resistor, which is thermal-conductively connected with the analyzed material. Temperature of the resistor was scanned by a semiconductive detector.

\section{Results and discussion}

\section{Influence of ferrite on cross-link density and structure}

Cross-link density and structure of the formed cross-links are important structural parameters of all rubber products determining their service properties. Not only the original properties but also their changes in time are dependent on these characteristics. Two different methods were used to determine cross-link density of the prepared composites. Chemical cross-link density, $\nu_{c h}$, was determined based on equilibrium swelling of samples in xylene and the total cross-link density, $\nu_{t}$, was determined based on deformation measurements of the prepared samples. In the first case, xylene diffuses into the samples and disrupts all physical interactions between the macromolecules of rubber. When equilibrium swelling is reached, the cross-link density is calculated by the Flory-Rehner equation (1). During the deformation measurements to a low deformation degree, the disruption of physical interactions between the macromolecules is not expected. As a result, concentration of all cross-links (chemical and physical) in the analyzed samples can be determined. From the difference between the total and chemical cross-link density, physical cross-link density, $\nu_{p h}$, can be calculated. Polymer-polymer physical interactions, polymer-filler physical interactions, also various intramolecular and intermolecular entanglements are involved in physical cross-link density.

From Fig. 1 it is evident that chemical cross-link density, $\nu_{c h}$, of composites based on pure NR shows

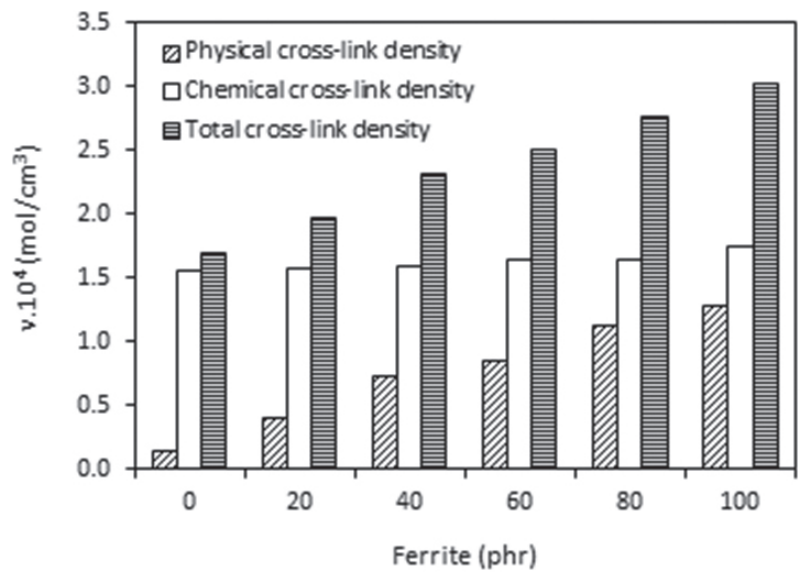

Fig. 2. Influence of ferrite content on physical $\nu_{p h}$, chemical $\nu_{c h}$ and total $\nu_{t}$ cross-link density of composites based on carbon black batch. 
a slightly increasing trend with the increasing amount of strontium ferrite. Physical cross-link density, which represents the difference between the total and the chemical cross-link density $\left(\nu_{t}\right.$ $-\nu_{c h}$ ), is much lower than $\nu_{c h}$ and also increases nonlinearly with the increasing content of the magnetic filler. The physical and chemical cross-link density of composites based on carbon black batch was higher compared to that of equivalent composites based on pure NR (Fig. 2). This can be attributed to the presence of carbon black which, as a reinforcing filler, contributes to the formation of chemical and mainly physical couplings in the rubber matrix. Thus, also the total cross-link density of composites based on NR batch is higher than the total crosslink density of composites based on pure NR.

An analysis revealed that the cross-link structure of both type composites was formed mainly from polysulfidic cross-links (about 50-60\%), the rest

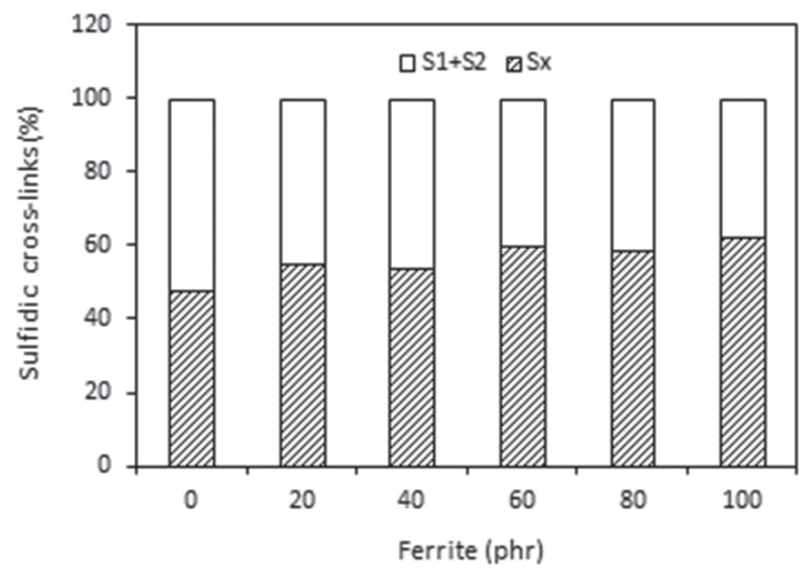

Fig. 3. Influence of ferrite content on the structure of sulfidic cross-links of composites based on NR

(S1 - monosulfidic cross-links,

S2 - disulfidic cross-links,

Sx - polysulfidic cross-links).

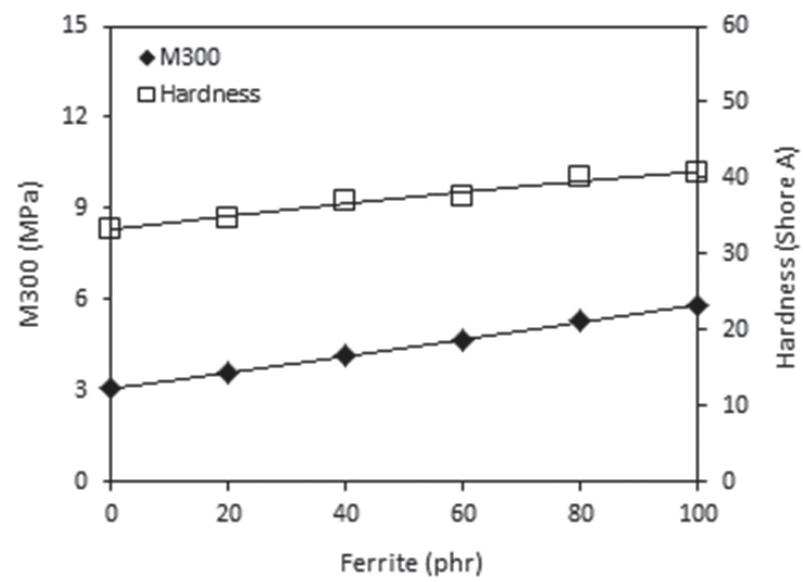

Fig. 5. Influence of ferrite content on modulus M300 and hardness of composites based on NR. cross-links are formed by mono- and disulfidic bridges (Figs. 3, 4). The achieved results are in line with general knowledge, according to which, in case semi-EV curing systems applied in cross-linking of rubber compounds, all three types of sulfidic cross-links are formed in the rubber matrix with dominance of polysulfidic cross-links. The amount of incorporated magnetic filler has no significant influence on the structure of the formed cross-links.

Influence of ferrite on physico-mechanical properties The incorporation of strontium ferrite into both composite types resulted in the increase of modulus M300 and hardness (Figs. 5, 6). The increase of modulus and hardness (in addition to the fact that hardness of strontium ferrite is much higher than that of the rubber matrix) can be attributed to the increase in cross-link density of both composite types. It can be also stated that higher cross-link density

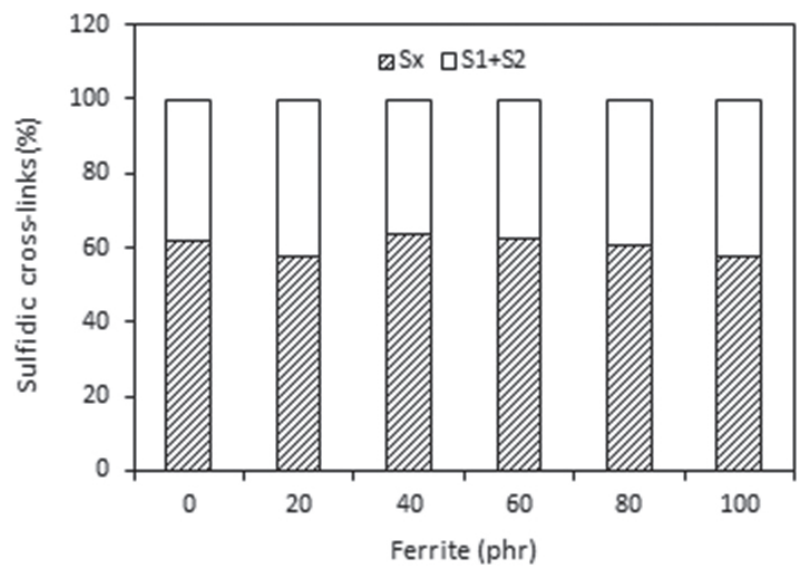

Fig. 4. Influence of ferrite content on the structure of sulfidic cross-links of composites based on carbon black batch (S1 - monosulfidic cross-links, $\mathrm{S} 2$ - disulfidic cross-links, $\mathrm{Sx}$ - polysulfidic cross-links).

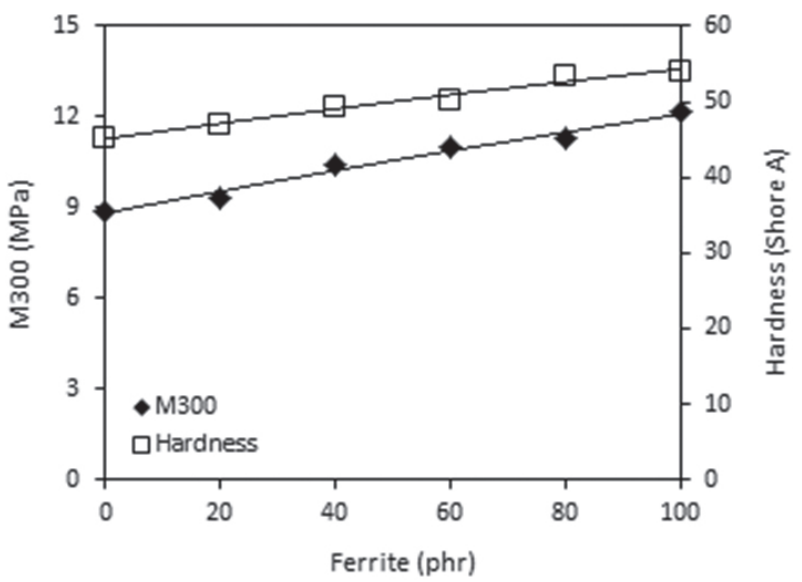

Fig. 6. Influence of ferrite content on modulus M300 and hardness of composites based on carbon black batch. 
and the presence of carbon black in the composites based on carbon black batch are responsible for higher values of these characteristics compared to equivalent composites based on pure NR.

On the other hand, the increase in cross-link density of both composite types with the increasing amount of strontium ferrite was responsible for the decrease of elongation at break (Fig. 7, 8). Again, higher cross-link density of composites based on carbon black batch was responsible for their lower elongation at break. The different character of tensile strength of both composite types in dependence on the strontium ferrite content was recorded. Incorporation of $40 \mathrm{phr}$ of strontium ferrite into the rubber matrix based on pure NR resulted in an enhancement of tensile strength by about $2 \mathrm{MPa}$ in comparison with the unfilled sample. Then, a slightly decreasing tendency of the tensile strength was recorded. As it can be seen in Fig. 7, the tensile strength of maximally filled composite reached the level of reference - unfilled sample. From Fig. 8 it is evident that the highest tensile strength of composites based on carbon black batch exhibited a reference sample filled only with carbon black. When ferrite was incorporated, the tensile strength decreased. Composite with maximum ferrite content showed the lowest tensile strength. Comparing both composite types, higher tensile strength was found in composites based on the carbon black batch. However, with the increasing amount of magnetic filler, the differences in tensile strength of both composite types became lower and the composites based on pure NR with 80 and 100 phr of ferrite already exhibited slightly higher tensile strength than equivalent composites based on the carbon black batch. This is interesting as in case of composites based on carbon black, much higher values of tensile strength were expected. Carbon black is a well-known reinforcing filler in rubber

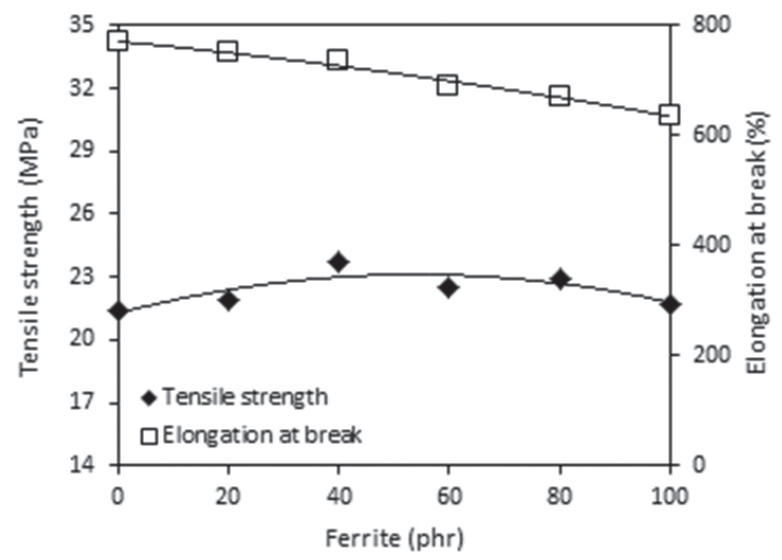

Fig. 7. Influence of ferrite content on tensile strength and elongation at break of composites based on NR. compounds. Therefore, higher tensile strength of composites based on pure NR with higher ferrite content is very surprising and it shows a promising aspect in the preparation of rubber composites with magnetic fillers. The results point out a slight reinforcing effect of strontium ferrite in the rubber matrix.

\section{Influence of ferrite on magnetic and thermo-physical characteristics}

The most important magnetic characteristics of all permanent magnets are the remanent magnetic induction, $B_{r}$, which represents the value of residual magnetism in the material when the external magnetic field is removed, and the coercive intensity of magnetic field (coercivity, $H_{c}$ ), which represents energy needed to abolish the remanent magnetic induction. The higher are the values of both characteristics, the better are the permanent magnets. Ferrites as hard magnetic materials have unique magnetic properties and their incorporation into rubber matrix should impart magnetic properties to the rubber composites as well. Therefore, the influence of strontium ferrite content on the magnetic properties of both composite types was investigated. As shown in Figs. 9, 10, the incorporation of ferrite resulted in a significant increase of remanent magnetic induction of both composite types. The remanent magnetic induction increased by more than $400 \%$ when the amount of strontium ferrite increased from minimum up to its maximum content. Slightly higher values of remanent magnetic induction were found for composites based on the carbon black batch. On the other hand, composites based on the carbon black batch exhibited lower coercivity compared with the equivalent composites based on NR. It is also apparent from Figs. 9, 10 that the coercivity of both composite types fluctuated only in the low range of experimental values

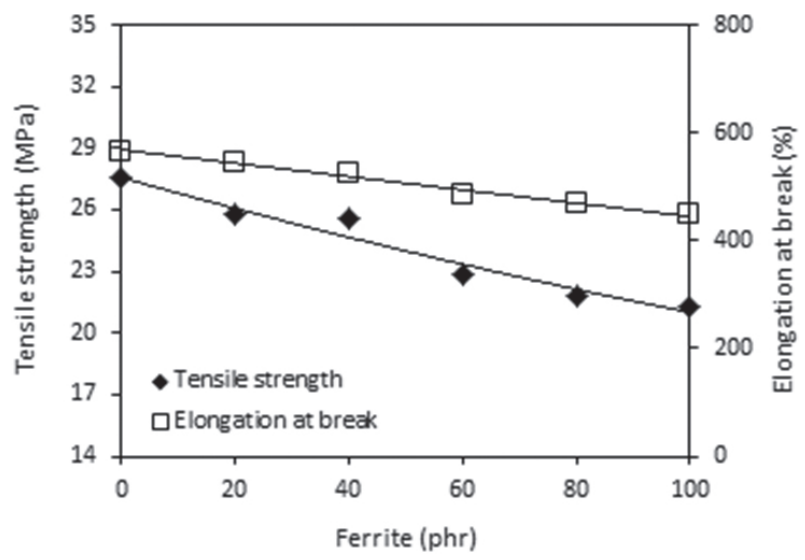

Fig. 8. Influence of ferrite content on tensile strength and elongation at break of composites based on carbon black batch. 


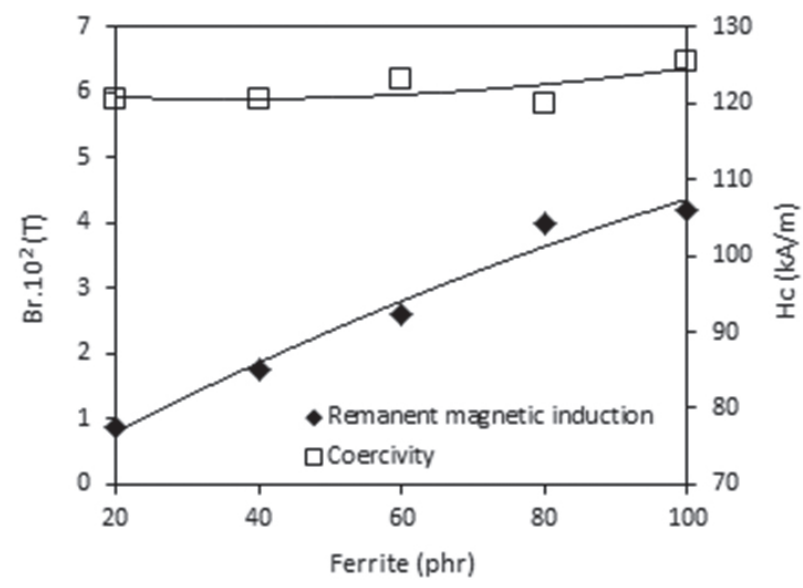

Fig. 9. Influence of ferrite content on remanent magnetic induction $B_{r}$ and coercivity $H_{c}$ of composites based on NR.

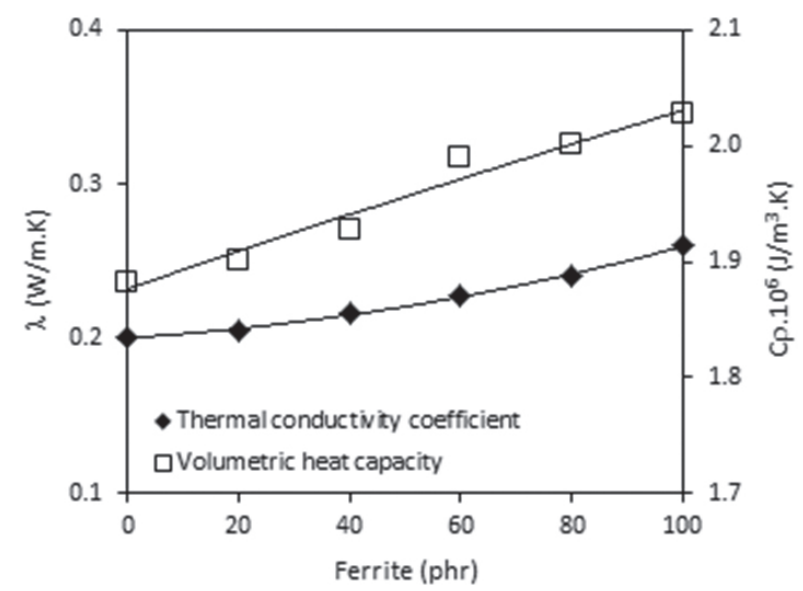

Fig. 11. Influence of ferrite content on thermal conductivity coefficient $\lambda$ and volumetric heat capacity $C \rho$ of composites based on NR.

almost independently on the amount of strontium ferrite.

Similarly, ferrites as metal oxide powders have higher thermal conductivity than elastomers and therefore their presence in rubber compounds should lead to increased thermal conductivity and thermal flow through the composites. Thus, the thermal conductivity coefficient, $\lambda$, and the volumetric heat capacity, $C \rho$, of rubber magnets were also examined. As expected, thermal conductivity coefficient and volumetric heat capacity of composites increased with the increasing amount of strontium ferrite although the increase of both parameters in dependence on the ferrite content was not very significant (Figs. 11, 12). Based on the achieved results it can be assumed that even at the maximum ferrite content in the rubber matrix, the percolation threshold of the filler is not reached and therefore the increase of thermal conductivity

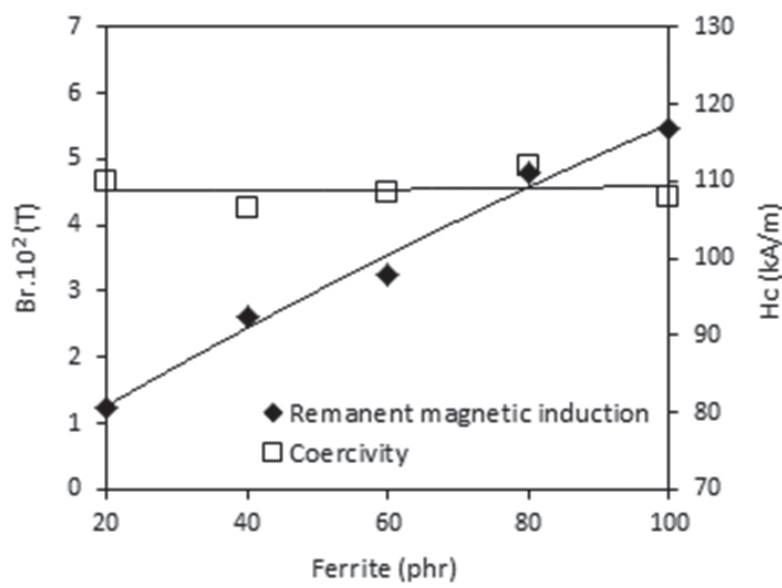

Fig. 10. Influence of ferrite content on remanent magnetic induction $B_{r}$ and coercivity $H_{c}$ of composites based on carbon black batch.

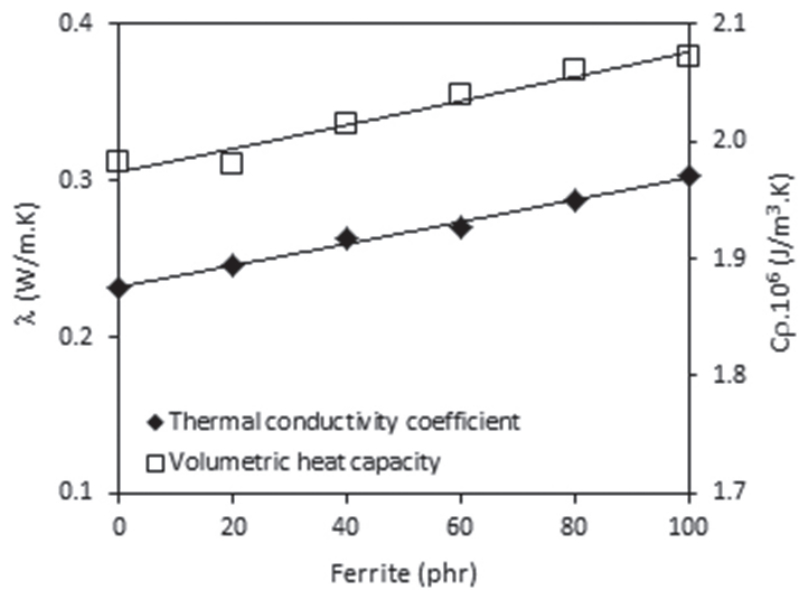

Fig. 12. Influence of ferrite content on thermal conductivity coefficient $\lambda$ and volumetric heat capacity $C \rho$ of composites based on carbon black batch.

is not substantial. When comparing both composite types, higher values of both parameters were found for composites based on carbon black batch. Thus, it can be concluded that carbon black also contributes to the increase of thermal conductivity of composite materials.

\section{Conclusion}

Rubber magnetic composites tested in this work were prepared by incorporation of strontium ferrite into rubber compounds based on pure NR and carbon black batch. The main aim was to investigate the influence of magnetic filler content on the cross-link density and properties of the prepared composites.

The results revealed that the incorporation of strontium ferrite leads to an increase of the crosslink density of composites, while the structure of 
the formed sulfidic cross-links remained almost unchanged with the change of the magnetic filler content. Dependences of modulus M300, hardness and elongation at break followed the those of crosslink density showing that the higher is the cross-link density, the higher is modulus and hardness and the lower is elongation at break. Tensile strength of composites based on carbon black batch showed a decreasing trend with the increasing content of magnetic filler, while positive effect of strontium ferrite was recorded in case of composites based on pure NR. This indicates a reinforcing effect of strontium ferrite on the rubber matrix. The remanent magnetic induction of both composite types significantly improved with the increasing amount of strontium ferrite. Incorporation of a magnetic filler into rubber matrices leads also to an increase of the thermo-physical characteristics of rubber magnets.

\section{Ackowledgement}

This work was supported by the Slovak Research and Development Agency under the contract No. APVV-160136.

\section{References}

Bellucci FS, de Almeida, FCL, Nobre MAL, Rodríguez-Pérez MA, Paschoalini AT, Job AE (2016) Magnetic properties of vulcanized natural rubber nanocomposites as a function of the concentration, size and shape of the magnetic fillers. Composites Part B. 85 : 196-206.

Das S, Nayak GC, Sahu SK, Routray PC, Roy AK, Baskey $\mathrm{H}$ (2015) Microwave absorption properties of doublelayer composites using CoZn/NiZn/MnZn-ferrite and titanium dioxide. Journal of Magnetism and Magnetic Materials. 377: 111-116.

Gutiérrez J, Martins P, Gonçalves R, Sencadas V, Lasheras A, Lanceros-Mendez S, Barandiarán JM (2015) Synthesis, physical and magnetic properties of $\mathrm{BaFe}_{12} \mathrm{O}_{19} / \mathrm{P}($ VDF-TrFE $)$ multifunctional composites. European Polymer Journal. 69: 224-231.

Hamed GR, editor (1992) Materials and Compounds: Engineering with Rubber. New York: Oxford University Press.

Jung HS, Kwon SH, Choi HJ, Jung JH, Kim YG (2016) Magnetic carbonyl iron/natural rubber composite elastomer and its magnetorheology. Composite Structures. 136: 106-112.

Kraus G (1963) Swelling of filler-reinforced vulcanizates. Journal of Applied Polymer Science. 7: 861-871.
Lechevallier L, LeBreton JM (2005) Substitution effects in M-type hexaferrite powders investigated by Mössbauer spectrometry. Journal of Magnetism and Magnetic Materials. 290-291: 1237-1239.

Li J, Zhang HF, Shao GQ, Chen D, Zhao GG, Gao ZS, Liu JH, Lu JS, Li XB (2015) Synthesis and properties of new multifunctional hexaferrite powders. Procedia Engineering. 102: 1885-1889.

Morrison NJ, Porter M (1984) Temperature effects on the stability of intermediates and crosslinks in sulfur vulcanization. Rubber Chemistry and Technology. 57: 63-86.

Namai A, Yoshikiyo M, Yamada K, Sakurai S, Goto T, Yoshida T, Miyazaki T, Nakajima M, Suemoto T, Tokoro H, Ohkoshi S. Hard magnetic ferrite with a gigantic coercivity and high frequency millimetre wave rotation. Nature Communications. DOI:10.1038/ ncomms2038.

Pattanayak R, Muduli R, Panda RK, Dash T, Sahu P, Raut S, Panigrahi S (2016) Investigating the effect of multiple grain-grain interfaces on electric and magnetic properties of $\left[50 \mathrm{wt} \% \mathrm{BaFe}_{12} \mathrm{O}_{19}-50\right.$ wt $\%$ $\left.\mathrm{Na}_{0.5} \mathrm{Bi}_{0.5} \mathrm{TiO}_{3}\right]$ composite system. Physica B. 485: $67-77$.

Pullar RC (2012) Hexagonal ferrites: A review of the synthesis, properties and applications of hexaferrite ceramics. Progress in Materials Science. 57: 1191-1334.

Saville B, Watson AA (1967) Structural characterization of sulfur-vulcanized rubber networks. Rubber Chemistry and Technology. 40: 100-149.

Sýkora R, Babayan V, Ušáková M, Kruželák J, Hudec I (2016) Rubber composite materials with the effects of electromagnetic shielding. Polymer Composites. 37(10): 2933-2939.

Tong, SY, Tung MJ, Ko WS, Huang YT, Wang YP, Wang LCh, Wu JM (2013) Effect of Ni fillers on microwave absorption and effective permeability of NiCuZn ferrite/ $\mathrm{Ni}$ / polymer functional composites. Journal of Alloys and Compounds. 550: 39-45.

Vinayasree S, Soloman MA, Sunny V, Mohanan P, Kurian P, Anantharaman MR (2016) A microwave absorber based on strontium ferrite-carbon black-nitril rubber for $\mathrm{S}$ and X-band applications. Composites Science and Technology. 82: 69-75.

Wang YF, Li QL, Zhang CR, Jing HX (2009) Preparation and magnetic properties of different morphology nano- $\mathrm{SrFe}_{12} \mathrm{O}_{19}$ particles prepared by sol-gel method. Journal of Alloys and Compounds. 467: 284-287.

Warner WC(1994) Methods of devulcanization. Rubber Chemistry and Technology. 67: 559-566.

Xie T, Xu L, Liu Ch, Ding S, Yang J, Wu W (2013) Synthesis and adsorption properties of high specific area strontium ferrite from Industrial Strontium Residue. Vacuum. 93: 71-78. 